\title{
Finite-Temperature Higgs Potentials
}

\author{
Dolgopolov M.V. ${ }^{1, \star}$, Gurskaya A.V. ${ }^{1, \star \star}$, and Rykova E.N. ${ }^{1, \star \star \star}$ \\ ${ }^{1}$ Samara University, Academician Pavlov 1, 443011 Samara, Russia.
}

\begin{abstract}
In the present article we consider the short description of the "FiniteTemperature Higgs Potentials" program for calculating loop integrals at vanishing external momenta and applications for extended Higgs potentials reconstructions. Here we collect the analytic forms of the relevant loop integrals for our work in reconstruction of the effective Higgs potential parameters in extended models (MSSM, NMSSM and etc.).
\end{abstract}

\section{Algebraic-Derivative recursion method}

The smallness of external state masses relative to the masses of loop particles allows us to take the limit where the external particles are massless. These integrals are particularly easy to solve using algebraic recursion relations or residue method (also remember [1]). But it is necessary to keep the external momentum $p_{\mu}$ general (i.e. nonzero) until it can be converted into an external mass in the amplitude, after which point one may take accurate limit $p \rightarrow 0$, especially in the cases with equivalent internal masses in loop.

In $d$ dimensions such two-point loop integral $B_{0}$ is defined as:

$$
\frac{1}{(4 \pi)^{2}} B_{0}\left(m^{2}, M^{2}\right)=\mu^{4-d} \int \frac{d^{d} k}{(2 \pi)^{d}} \frac{i}{\left(k^{2}-m^{2}\right)\left[k^{2}-M^{2}\right]} .
$$

In order to derive asymptotic results we expand $B_{0}\left(p^{2}, m_{1}^{2}, m_{2}^{2}\right)$ in powers of the external momentum $p^{2}$ :

$$
B_{0}\left(p^{2}, m_{1}^{2}, m_{2}^{2}\right)=-\Delta_{\mu}-\frac{m_{1}^{2} \log m_{1}^{2}-m_{2}^{2} \log m_{2}^{2}}{m_{2}^{2}-m_{1}^{2}}+O\left(\frac{p^{2}}{m_{1}^{2}+m_{2}^{2}}\right) .
$$

The 3- and 4-point loop integrals at vanishing external momenta are defined as:

$$
\begin{aligned}
\frac{1}{(4 \pi)^{2}} C_{2 n}\left(m_{1}^{2}, m_{2}^{2}, m_{3}^{2}\right) & =\mu^{4-d} \int \frac{d^{d} k}{(2 \pi)^{d}} \frac{i k^{2 n}}{\prod_{i}^{3}\left(k^{2}-m_{i}^{2}\right)}, \\
\frac{1}{(4 \pi)^{2}} D_{2 n}\left(m_{1}^{2}, m_{2}^{2}, m_{3}^{2}, m_{4}^{2}\right) & =\mu^{4-d} \int \frac{d^{d} k}{(2 \pi)^{d}} \frac{i k^{2 n}}{\prod_{i}^{4}\left(k^{2}-m_{i}^{2}\right)} .
\end{aligned}
$$

We follow the 'Buras' convention of Appendix A.5 in [2], and [3] with some extent, corrections and prove. Note that the above equations are manifestly equivalent under interchange of the arguments

\footnotetext{
${ }^{\star}$ Dolgopolov Mikhail Vyacheslavovich, e-mail: mvdolg@yandex.ru

$\star \star$ Gurskaya Albina Valentinovna, e-mail: a-gurska@yandex.ru

$\star \star \star$ Rykova Elza Nurovna, emailelzarykova@rambler.ru
} 
and explicit calculations show that one may reshuffle the order of the arguments without changing the result (for functions with unequal arguments it is obvious, and for functions with the equivalent arguments it demands accuracy!).

The two-point loop integral in tensor reduction $B_{1}$ can be defined as:

$$
\frac{1}{(4 \pi)^{2}} p_{\mu} B_{1}\left(p, m^{2}, M^{2}\right)=\mu^{4-d} \int \frac{d^{d} k}{(2 \pi)^{d}} \frac{i k_{\mu}}{\left(k^{2}-m^{2}\right)\left[(k+p)^{2}-M^{2}\right]}
$$

Such integrals appear in the self-energy contributions.

It turns out that this is very simple to evaluate integrals (1), (2), (3) with the following algebraic relations:

$$
\begin{gathered}
\frac{1}{\left(k^{2}-m_{1}^{2}\right)\left(k^{2}-m_{2}^{2}\right)}=\frac{1}{m_{1}^{2}-m_{2}^{2}}\left(\frac{1}{k^{2}-m_{1}^{2}}-\frac{1}{k^{2}-m_{2}^{2}}\right), \\
\frac{k^{2}}{k^{2}-m^{2}}=1+\frac{m^{2}}{k^{2}-m^{2}} .
\end{gathered}
$$

Note that denominators of integrals under consideration are spherically symmetric. And for the case of all equivalent masses the particularly well-known general integral can be performed using $\Gamma$ function:

$$
\int \frac{d^{n} k}{(2 \pi)^{n}} \frac{\left(k^{2}\right)^{b}}{\left(k^{2}-A^{2}\right)^{a}}=\frac{i}{(4 \pi)^{n / 2}}(-1)^{a+b}\left(A^{2}\right)^{b-a+n / 2} \frac{\Gamma(b+n / 2) \Gamma(-b+a-n / 2)}{\Gamma(n / 2) \Gamma(a)},
$$

where the left-hand side is an integral in n-dimensional Minkowski space. In the private case at $b=0$

$$
\int \frac{d^{n} k}{(2 \pi)^{n}} \frac{1}{\left(k^{2}-A^{2}\right)^{a}}=\frac{i}{(4 \pi)^{n / 2}}(-1)^{a}\left(A^{2}\right)^{-a+n / 2} \frac{\Gamma(a-n / 2)}{\Gamma(a)} .
$$

\subsection{Results for one-loop scalar integrals}

The explicit formulae at $d \rightarrow 4$ are listed below (we give also expressions for some 3-point functions proportional to higher momenta powers, may be useful in contributions for effective Higgs potential 
calculations [4-6]) and may be represented only in terms of $B_{0}$ integral, or $A_{0}(8)$ :

$$
\begin{aligned}
& \frac{1}{(4 \pi)^{2}} A_{0}\left(m^{2}\right)=\mu^{4-d} \int \frac{d^{d} k}{(2 \pi)^{d}} \frac{i}{k^{2}-m^{2}}=i \frac{\mu^{4-d} i}{(4 \pi)^{d / 2}}(-1)\left(m^{2}\right)^{-1+d / 2} \Gamma(1-d / 2)= \\
& =\frac{-i i}{16 \pi^{2}}\left(1+\frac{\varepsilon}{2} \log \left(4 \pi \mu^{2}\right)+O\left(\varepsilon^{2}\right)\right) m^{2}\left(1-\frac{\varepsilon}{2} \log \left(m^{2}\right)+O\left(\varepsilon^{2}\right)\right) \times \\
& \times \quad(-1)\left(\frac{2}{\varepsilon}+1-\gamma_{E}+O(\varepsilon)\right) \Rightarrow \\
& \Rightarrow \quad A_{0}(x)=x\left(-\Delta_{\mu}+\log x\right) \text {, } \\
& B_{0}(x, y)=\frac{1}{x-y}\left[A_{0}(x)-A_{0}(y)\right]=\frac{-1}{x-y}\left[x \Delta_{\mu}-y \Delta_{\mu}-x \log x+y \log y\right]= \\
& =-\Delta_{\mu}-\frac{-x \log x+y \log x-y \log x+y \log y}{x-y}= \\
& =-\Delta+\log \frac{x}{\mu^{2}}-\frac{y}{x-y} \log \frac{y}{x}= \\
& =B_{0}(y, x)=-\Delta+\log \frac{y}{\mu^{2}}-\frac{x}{y-x} \log \frac{x}{y}, \\
& C_{0}(x, y, z)=\frac{1}{y-z}\left[B_{0}(x, y)-B_{0}(x, z)\right]=\frac{y \log \frac{y}{x}}{(x-y)(z-y)}+\frac{z \log \frac{z}{x}}{(x-z)(y-z)}, \\
& C_{0}(x, y, y)=\frac{\partial}{\partial y} B_{0}(x, y)=\frac{1}{y-x}\left[1+\frac{x \log \frac{x}{y}}{y-x}\right], \\
& C_{2}(x, y, z)=B_{0}(x, y)+{ }_{z} C_{0}(x, y, z)= \\
& =-\Delta-\log \frac{\mu^{2}}{x}+\frac{y^{2} \log \frac{y}{x}}{(x-y)(z-y)}+\frac{z^{2} \log \frac{z}{x}}{(x-z)(y-z)}, \\
& C_{2}(x, y, y)=B_{0}(y, x)+y C_{0}(x, y, y)= \\
& =-\Delta_{\mu}+\log y-\frac{x}{y-x} \log \frac{x}{y}+\frac{y}{y-x}\left[1+\frac{x \log \frac{x}{y}}{y-x}\right]= \\
& =-\Delta-\log \frac{\mu^{2}}{y}-\frac{x}{x-y}\left[\frac{y}{x}-\frac{x \log \frac{x}{y}}{x-y}\right]= \\
& =B_{0}(x, y)+y C_{0}(x, y, y)= \\
& =-\Delta-\log \frac{\mu^{2}}{x}+\frac{y}{y-x}\left[1-\frac{(y-2 x) \log \frac{x}{y}}{y-x}\right] \text {, } \\
& =\frac{\partial}{\partial y} A_{0}(y)+x C_{0}(x, y, y)= \\
& =-\Delta-\log \frac{\mu^{2}}{y}-\frac{x}{x-y}\left[\frac{y}{x}-\frac{x \log \frac{x}{y}}{x-y}\right] \text {. }
\end{aligned}
$$


The answers (16), (18), and (17) are identical indeed, check by Mathematica is done. Check for last equations:

$$
\begin{aligned}
& \frac{1}{(4 \pi)^{2}} \frac{\partial}{\partial m^{2}} A_{0}\left(m^{2}\right)=\mu^{4-d} \int \frac{d^{d} k}{(2 \pi)^{d}} \frac{i}{\left(k^{2}-m^{2}\right)^{2}}=i \frac{\mu^{4-d} i}{(4 \pi)^{d / 2}}(-1)^{2}\left(m^{2}\right)^{-2+d / 2} \Gamma(2-d / 2)=(19) \\
&=\frac{i i}{16 \pi^{2}}\left(1+\frac{\varepsilon}{2} \log \left(4 \pi \mu^{2}\right)+O\left(\varepsilon^{2}\right)\right)\left(1-\frac{\varepsilon}{2} \log \left(m^{2}\right)+O\left(\varepsilon^{2}\right)\right) \times \\
& \times\left(\frac{2}{\varepsilon}-\gamma_{E}+O(\varepsilon)\right)=\left(-\Delta_{\mu}+1\right)+\log x \\
& \text { yes } \quad \frac{\partial}{\partial x} A_{0}(x)=-\Delta_{\mu}+\log x+1 .
\end{aligned}
$$

Equal arguments correspond to equivalent masses. The divergent part is

$$
\Delta_{\{\mu ; 1\}}=\frac{2}{4-d}+\log \left(4 \pi\left\{\mu^{2} ; 1\right\}\right)-\gamma_{\mathrm{E}}+1
$$

where $\mu$ is the renormalization scale.

And

$$
\begin{aligned}
& C_{11}(x, y)=-\frac{x-3 y}{4(x-y)^{2}}+\frac{y^{2}}{2(x-y)^{3}} \log \frac{y}{x}, \\
& C_{12}(x, y)=-\frac{x+y}{2(x-y)^{2}}-\frac{x y}{3(x-y)^{3}} \log \frac{y}{x} .
\end{aligned}
$$

The explicit formulae for the four-point integrals at vanishing external momenta are (possible cross-checks are provided):

$$
\begin{aligned}
& D_{0}(x, y, z, t)=\frac{1}{z-t}\left(C_{0}(x, y, z)-C_{0}(x, y, t)\right)= \\
& =\frac{y \log \frac{y}{x}}{(y-x)(y-z)(y-t)}+\frac{z \log \frac{z}{x}}{(z-x)(z-y)(z-t)}+\frac{t \log \frac{t}{x}}{(t-x)(t-y)(t-z)}, \\
& D_{0}(x, y, z, z)=\frac{\partial}{\partial z} C_{0}(z, y, x)=\frac{\partial}{\partial z}\left(\frac{y \log \frac{y}{z}}{(z-y)(x-y)}+\frac{x \log \frac{x}{z}}{(z-x)(y-x)}\right)= \\
& =\frac{1}{(x-z)(y-z)}+\frac{x \log \frac{x}{z}}{(x-y)(x-z)^{2}}+\frac{y \log \frac{y}{z}}{(y-x)(y-z)^{2}} \text {, } \\
& D_{2}(x, y, z, t)=C_{0}(x, y, z)+t D_{0}(x, y, z, t)= \\
& =\frac{y^{2} \log \frac{y}{x}}{(y-x)(y-z)(y-t)}+\frac{z^{2} \log \frac{z}{x}}{(z-x)(z-y)(z-t)}+\frac{t^{2} \log \frac{t}{x}}{(t-x)(t-y)(t-z)},
\end{aligned}
$$




$$
\begin{aligned}
& D_{2}(0, x, y, z)=\frac{1}{y-x}\left(B_{0}(z, y)-B_{0}(z, x)\right)=-\frac{1}{y-x}\left(\frac{x \log \frac{x}{z}}{x-z}-\frac{y \log \frac{y}{z}}{y-z}\right), \\
& D_{2}(0, x, x, y)=C_{0}(y, x, x)=-\frac{1}{y-x}\left(1-\frac{y \log \frac{y}{x}}{y-x}\right), \\
& D_{2}(x, y, z, z)=\frac{\partial}{\partial z} C_{2}(z, y, x)=\frac{z}{(x-z)(y-z)}+\frac{x^{2} \log \frac{x}{z}}{(x-y)(x-z)^{2}}+\frac{y^{2} \log \frac{y}{z}}{(y-x)(y-z)^{2}} .
\end{aligned}
$$

A null argument represents the negligible mass of the virtual particle.

The case with two pairs of equivalent masses in $D_{0}$ can be reduced with the following algebraic relation:

$$
\frac{1}{\left(k^{2}-A^{2}\right)^{2}\left(k^{2}-B^{2}\right)^{2}}=\frac{1}{\left(A^{2}-B^{2}\right)^{2}}\left(\frac{1}{\left(k^{2}-A^{2}\right)^{2}}+\frac{1}{\left(k^{2}-B^{2}\right)^{2}}\right)-\frac{2}{\left(A^{2}-B^{2}\right)^{3}}\left(\frac{1}{k^{2}-A^{2}}-\frac{1}{k^{2}-B^{2}}\right),
$$

or by derivatives:

$$
D_{0}(x, x, y, y)=\frac{\partial^{2}}{\partial x \partial y} B_{0}(x, y)=\frac{\partial}{\partial x} C_{0}(x, y, y)=\frac{2}{(x-y)^{2}}+\frac{x+y}{(y-x)^{3}} \log \frac{x}{y} .
$$

The explicit formula for the 2-point loop integral $B_{1}$ at vanishing external momentum is:

$$
B_{1}(0, x, y)=\frac{1}{2} B_{0}(0, x, y)-\frac{1}{4} \frac{x+y}{x-y}+\frac{1}{2} \frac{x y}{(x-y)^{2}} \log \frac{x}{y}=\frac{1}{4}+\frac{1}{2} C_{2}(x, y, y),
$$

where $C_{2}(x, y, y)$ is given in eq. (16) or (17) or equivalently (18).

And some additional private checks:

$$
\begin{gathered}
B_{0}\left(m^{2}\right)=\frac{\partial}{\partial m^{2}} A_{0}\left(m^{2}\right)=-\Delta_{\mu}+\log m^{2}+1, \\
C_{0}\left(m^{2}\right)=\frac{1}{2} \frac{\partial}{\partial m^{2}} B_{0}\left(m^{2}\right)=\frac{1}{2 m^{2}}, \\
D_{0}\left(m^{2}\right)=\frac{1}{3} \frac{\partial}{\partial m^{2}} C_{0}\left(m^{2}\right)=-\frac{1}{6 m^{4}} .
\end{gathered}
$$

\section{Finite temperature one-loop integrals}

In the finite temperature field theory Feynman diagrams with boson propagators, containing Matsubara frequencies $\omega_{n}=2 \pi n T(n=0, \pm 1, \pm 2, \ldots)$, lead to structures of the form

$$
I\left[m_{1}, m_{2}, \ldots, m_{b}\right]=T \sum_{n=-\infty}^{\infty} \int \frac{d \mathbf{k}}{(2 \pi)^{3}} \prod_{i=1}^{b} \frac{(-1)^{b}}{\left(\mathbf{k}^{2}+\omega_{n}^{2}+m_{j}^{2}\right)},
$$

Here $\mathbf{k}$ is the three-dimensional momentum in a system with the temperature $T$. In the following calculations first we perform integration with respect to $\mathbf{k}$ and then take the sum, using the reduction 
to three-dimensional theory in the high-temperature limit for zero frequencies. At $n \neq 0$ the result is $[7,8]$

$$
I\left[m_{1}, m_{2}, \ldots, m_{b}\right]=2 T(2 \pi T)^{3-2 b} \frac{(-1)^{b} \pi^{3 / 2}}{(2 \pi)^{3}} \frac{\Gamma(b-3 / 2)}{\Gamma(b)} S(M, b-3 / 2),
$$

where

$$
S(M, b-3 / 2)=\int\{d x\} \sum_{n=1}^{\infty} \frac{1}{\left(n^{2}+M^{2}\right)^{b-3 / 2}}, \quad M^{2} \equiv\left(\frac{m}{2 \pi T}\right)^{2} .
$$

For $b>1$ the parameter $m^{2}$ is a linear function dependent on $m_{i}^{2}$ and the variables $\{d x\}$ of Feynman parametrization, which are the integration variables in (37). At the integer values of $b$ the integrand in (37) is a generalized Hurwitz zeta-function $[9,10]$. Note that for the leading threshold corrections to effective parameters of the two-doublet potential $b>2$, so the wave-function renormalization appears in connection with the divergence at $b=2$ (which is suppressed by vertex factors, see [11, 12]).

A number of integrals can be easily calculated. We calculate the integral

$$
J_{0}\left[a_{1}, a_{2}\right]=\int \frac{d \mathbf{k}}{(2 \pi)^{3}} \frac{1}{\left(\mathbf{k}^{2}+a_{1}^{2}\right)\left(\mathbf{k}^{2}+a_{2}^{2}\right)}=\frac{1}{4 \pi\left(a_{1}+a_{2}\right)},
$$

taking a residue in the spherical coordinate system. Here $a_{1 ; 2}^{2}$ are the sums of squared frequency and squared mass, see (35). Derivatives of $J_{0}$ with respect to $a_{1}$ and $a_{2}$ can be used for calculation of integrals

$$
\begin{gathered}
J_{1}\left[a_{1}, a_{2}\right]=\int \frac{d \mathbf{k}}{(2 \pi)^{3}} \frac{1}{\left(\mathbf{k}^{2}+a_{1}^{2}\right)^{2}\left(\mathbf{k}^{2}+a_{2}^{2}\right)}=-\frac{1}{2 a_{1}} \frac{\partial J_{0}}{\partial a_{1}}=\frac{1}{8 \pi a_{1}\left(a_{1}+a_{2}\right)^{2}}, \\
J_{2}\left[a_{1}, a_{2}\right]=\int \frac{d \mathbf{k}}{(2 \pi)^{3}} \frac{1}{\left(\mathbf{k}^{2}+a_{1}^{2}\right)^{2}\left(\mathbf{k}^{2}+a_{2}^{2}\right)^{2}}=\frac{1}{4 a_{1} a_{2}} \frac{\partial^{2} J_{0}}{\partial a_{1} \partial a_{2}}=\frac{1}{8 \pi a_{1} a_{2}\left(a_{1}+a_{2}\right)^{3}} .
\end{gathered}
$$

and ${ }^{1}$

$$
\begin{aligned}
& J_{3}\left[a_{1}, a_{2}, a_{3}\right]=\int \frac{d \mathbf{k}}{(2 \pi)^{3}} \frac{1}{\left(\mathbf{k}^{2}+a_{1}^{2}\right)\left(\mathbf{k}^{2}+a_{2}^{2}\right)\left(\mathbf{k}^{2}+a_{3}^{2}\right)}=\frac{1}{4 \pi\left(a_{1}+a_{2}\right)\left(a_{1}+a_{3}\right)\left(a_{2}+a_{3}\right)}, \\
& J_{4}\left[a_{1}, a_{2}, a_{3}\right]=\int \frac{d \mathbf{k}}{(2 \pi)^{3}} \frac{1}{\left(\mathbf{k}^{2}+a_{1}^{2}\right)^{2}\left(\mathbf{k}^{2}+a_{2}^{2}\right)\left(\mathbf{k}^{2}+a_{3}^{2}\right)}=\frac{2 a_{1}+a_{2}+a_{3}}{8 \pi a_{1}\left(a_{1}+a_{2}\right)^{2}\left(a_{1}+a_{3}\right)^{2}\left(a_{2}+a_{3}\right)},
\end{aligned}
$$

Thus, the procedure of Feynman parametrization is not used. Substituting $a_{1}^{2}=4 \pi^{2} n^{2} T^{2}+m_{1}^{2}$ and $a_{2}^{2}=4 \pi^{2} n^{2} T^{2}+m_{2}^{2}$ to (38) and taking the sum over Matsubara frequencies after the integration we get

$$
I_{0}\left[m_{1}, m_{2}\right]=\sum_{n=-\infty, n \neq 0}^{\infty} J_{0}^{n}\left[m_{1}, m_{2}\right]=\sum_{n=-\infty, n \neq 0}^{\infty} \frac{1}{4 \pi\left(\sqrt{4 \pi^{2} n^{2} T^{2}+m_{1}^{2}}+\sqrt{4 \pi^{2} n^{2} T^{2}+m_{2}^{2}}\right)} .
$$

or, after redefinition of mass parameters $M_{1 ; 2}=m_{1 ; 2} / 2 \pi T$ the temperature corrections to effective potential are expressed by summed integrals

$$
I_{1}\left[M_{1}, M_{2}\right]=-\frac{1}{64 \pi^{4} T^{2}} \sum_{n=-\infty, n \neq 0}^{\infty} \frac{1}{\sqrt{M_{1}^{2}+n^{2}}\left(\sqrt{M_{1}^{2}+n^{2}}+\sqrt{M_{2}^{2}+n^{2}}\right)^{2}},
$$

\footnotetext{
${ }^{1}$ The same results for $J_{3}$ and $J_{4}$ can be found in $[14]$ and $[15,16]$, where they appear in the context of high temperature dimensional reduction.
} 


$$
I_{2}\left[M_{1}, M_{2}\right]=\frac{1}{256 \pi^{5} T^{4}} \sum_{n=-\infty, n \neq 0}^{\infty} \frac{1}{\sqrt{M_{1}^{2}+n^{2}} \sqrt{M_{2}^{2}+n^{2}}\left(\sqrt{M_{1}^{2}+n^{2}}+\sqrt{M_{2}^{2}+n^{2}}\right)^{3}} .
$$

Note that the series (43) are divergent, but the derivatives (44) and (45) are convergent for all $M_{1 ; 2}$. In the following it will be convenient to keep separately the terms for zero and nonzero modes in the sum. Both terms will be temperature-dependent since the zero-mode integrals coincide with (38)-(40), where $a_{i}^{2}=m_{i}^{2}$ and the factor $T$ should be accounted for. Numerical check of the zero temperature limiting case $T \rightarrow 0$ demonstrates that the non-temperature field theory results are successfully reproduced. In the high-temperature limit the zero mode gives dominant contribution in agreement with a known suppression of quantum effects at increasing temperatures.

The sum of integrals (44) and (45) can be expressed by means of the generalized zeta-function. Such forms can be derived if we introduce Feynman parameters in the integrand of (38)

$$
\frac{1}{\left[\mathbf{k}^{2}+m_{a}^{2}\right]\left[\mathbf{k}^{2}+m_{b}^{2}\right]}=\int_{0}^{1} \frac{d x}{\left(\left[\mathbf{k}^{2}+m_{a}^{2}\right] x+\left[\mathbf{k}^{2}+m_{b}^{2}\right](1-x)\right)^{2}},
$$

and redefine $\mathbf{k} \longrightarrow \mathbf{p}=\mathbf{k} / 2 \pi T, M^{2}\left(M_{a}, M_{b}, x\right)=\left(M_{a}^{2}-M_{b}^{2}\right) x+M_{b}^{2}$. Then we get

$$
\frac{1}{\left[\mathbf{k}^{2}+m_{a}^{2}\right]\left[\mathbf{k}^{2}+m_{b}^{2}\right]}=\frac{1}{(2 \pi T)^{4}} \int_{0}^{1} \frac{d x}{\left(\mathbf{p}^{2}+n^{2}+M^{2}\right)^{2}} \text {. }
$$

and divergent series for $(38)\left(d \mathbf{k}=(2 \pi T)^{3} d \mathbf{p}\right)$

$$
I_{0}\left[M_{a}, M_{b}\right]=\frac{1}{2 \pi T} \int_{0}^{1} d x \sum_{n=-\infty, n \neq 0}^{\infty} \int \frac{d \mathbf{p}}{(2 \pi)^{3}} \frac{1}{\left(\mathbf{p}^{2}+n^{2}+M^{2}\right)^{2}},
$$

With the help of dimensional regularization or differentiating the integral

$$
\int \frac{d \mathbf{p}}{(2 \pi)^{3}} \frac{1}{\left(\mathbf{p}^{2}+M^{2}\right)}=-\frac{M}{4 \pi}+O\left(\frac{M^{2}}{T^{2}}\right)
$$

over the parameter $M$, the equation (48) can be reduced to

$$
I_{0}\left[M_{a}, M_{b}\right]=\frac{1}{16 \pi^{2} T} \int_{0}^{1} d x \zeta\left(2, \frac{1}{2}, M^{2}\right),
$$

where $\zeta(u, s, t)$ is the generalized Hurwitz zeta-function $[9,10]:{ }^{2}$

$$
\zeta(u, s, t)=\sum_{n=1}^{\infty} \frac{1}{\left(n^{u}+t\right)^{s}} .
$$

So in the case under consideration the sums of integrals (44) and (45) can be calculated by differentiation of (50) with respect to mass parameters participating in $M=M\left(M_{a}, M_{b}, x\right)$. Differentiation increases the power $s$ in the denominator of (50) giving convergent integrals

$$
\begin{gathered}
I_{1}\left[M_{a}, M_{b}\right]=\frac{T}{2 M_{a}} \frac{\partial}{\partial M_{a}} I_{0}=-\frac{1}{64 \pi^{4} T^{2}} \int_{0}^{1} d x x \zeta\left[2, \frac{3}{2}, M^{2}(x)\right], \\
I_{2}\left[M_{a}, M_{b}\right]=-\frac{1}{2 M_{b}} \frac{\partial}{\partial M_{b}}\left(-I_{1}\right)=\frac{3}{256 \pi^{6} T^{4}} \int_{0}^{1} d x x(1-x) \zeta\left[2, \frac{5}{2}, M^{2}(x)\right] .
\end{gathered}
$$

The integrals (52) and (53) are equal to the series (44) and (45), respectively.

\footnotetext{
${ }^{2}$ Note that (non-generalized) Hurwitz zeta-function is defined by $\zeta(s, t)=\sum_{n=0}^{\infty} \frac{1}{[n+t]^{s}}$.
} 


\section{Examples. Results with one-loop contributions}

\section{Two pairs of different virtual masses}

Four-point function with pairs of equivalent virtual masses:

$$
\underline{J_{2}\left(m_{1}, m_{2}\right)}=i \operatorname{Tr} \int \frac{d^{4} k}{(2 \pi)^{4}} \frac{i^{4}}{\left(\hat{k}-m_{1}\right)\left(\hat{k}-m_{2}\right)\left(\hat{k}-m_{1}\right)\left(\hat{k}-m_{2}\right)}=
$$

$=4 \frac{1}{16 \pi^{2}} B_{0}\left(m_{1}^{2}, m_{1}^{2}\right)+4\left(m_{1}^{2}+3 m_{2}^{2}+4 m_{1} m_{2}\right) \frac{1}{16 \pi^{2}} C_{0}\left(m_{2}^{2}, m_{1}^{2}, m_{1}^{2}\right)+8 m_{2}^{2}\left(m_{2}+m_{1}\right)^{2} \frac{1}{16 \pi^{2}} D_{0}\left(m_{1}^{2}, m_{1}^{2}, m_{2}^{2}, m_{2}^{2}\right)$.

This result (3) have to be symmetric under interchange $m_{1} \rightleftarrows m_{2}$. Check for equivalent virtual masses:

$$
\lim _{m_{2} \rightarrow m_{1}} J_{2}\left(m_{1}, m_{2}\right)=J_{2}(m)=\frac{1}{16 \pi^{2}}\left(-4 \Delta_{\mu}+4 \log m^{2}+4+16-\frac{16}{3}\right)
$$

coincides.

\section{Three different virtual masses}

$$
\begin{aligned}
& \underline{J_{3}\left(m_{1}, m_{2}, m_{3}\right)}=i \operatorname{Tr} \int \frac{d^{4} k}{(2 \pi)^{4}} \frac{i^{4}}{\left(\hat{k}-m_{1}\right)\left(\hat{k}-m_{2}\right)\left(\hat{k}-m_{1}\right)\left(\hat{k}-m_{3}\right)}= \\
& =4 \frac{1}{16 \pi^{2}} B_{0}\left(m_{1}^{2}, m_{1}^{2}\right)+4\left(3 m_{1}^{2}+m_{2} m_{3}+2 m_{1}\left(m_{2}+m_{3}\right)\right) \frac{1}{16 \pi^{2}} C_{0}\left(m_{1}^{2}, m_{2}^{2}, m_{3}^{2}\right)+ \\
& +8 m_{1}^{2}\left(m_{1}^{2}+m_{1}\left(m_{2}+m_{3}\right)+m_{2} m_{3}\right) \frac{1}{16 \pi^{2}} D_{0}\left(m_{1}^{2}, m_{1}^{2}, m_{2}^{2}, m_{3}^{2}\right) .
\end{aligned}
$$

The selfcheck for last is $\lim _{m_{3} \rightarrow m_{2}} J_{3}\left(m_{1}, m_{2}, m_{3}\right)=J_{2}\left(m_{1}, m_{2}\right)$ is fulfilled.

\section{Four different virtual masses}

$$
\begin{gathered}
\frac{J_{4}\left(m_{1}, m_{2}, m_{3}, m_{4}\right)}{=}=i \operatorname{Tr} \int \frac{d^{4} k}{(2 \pi)^{4}} \frac{i^{4}}{\left(\hat{k}-m_{1}\right)\left(\hat{k}-m_{2}\right)\left(\hat{k}-m_{3}\right)\left(\hat{k}-m_{4}\right)}= \\
=4 \frac{1}{16 \pi^{2}} B_{0}\left(m_{3}^{2}, m_{4}^{2}\right)+4 m_{1}^{2} \frac{1}{16 \pi^{2}} C_{0}\left(m_{1}^{2}, m_{3}^{2}, m_{4}^{2}\right)+ \\
+4\left(m_{2}^{2}+m_{1} m_{2}+\left(m_{1}+m_{2}\right)\left(m_{3}+m_{4}\right)+m_{3} m_{4}\right) \frac{1}{16 \pi^{2}} C_{0}\left(m_{2}^{2}, m_{3}^{2}, m_{4}^{2}\right)+ \\
\left.+4\left\{m_{2}^{2}\left[m_{1}^{2}+m_{1} m_{2}+\left(m_{1}+m_{2}\right)\left(m_{3}+m_{4}\right)+m_{3} m_{4}\right)\right]+m_{1} m_{2} m_{3} m_{4}\right\} \frac{1}{16 \pi^{2}} D_{0}\left(m_{1}^{2}, m_{2}^{2}, m_{3}^{2}, m_{4}^{2}\right) .
\end{gathered}
$$

The self-checks are interchange symmetry between parameters and again $\lim _{m_{4} \rightarrow m_{3}} J_{4}\left(m_{1}, m_{2}, m_{3}, m_{4}\right)=$ $J_{3}\left(m_{1}, m_{2}, m_{3}\right)$ and etc.

The common principal check is also the contraction of fermion and boson loops diverging parts.

Note, that the integrals $J_{2}, J_{3}$ can be rewritten in terms of $J_{4}$, which is expressed in terms of one integral $A_{0}$ (or $B_{0}$ ) with the help of Mathematica program.

\section{Four equivalent virtual masses}

$$
\underline{J_{1}(m)}=i \operatorname{Tr} \int \frac{d^{4} k}{(2 \pi)^{4}} \frac{i^{4}}{(\hat{k}-m)(\hat{k}-m)(\hat{k}-m)(\hat{k}-m)}=\frac{1}{16 \pi^{2}}\left(-4 \Delta+4 \log \frac{m^{2}}{\mu^{2}}+\frac{44}{3}\right) .
$$




\subsection{One-loop gaugino-higgsino contributions to $\lambda_{1}$}

The couplings to be taken into account are the following:
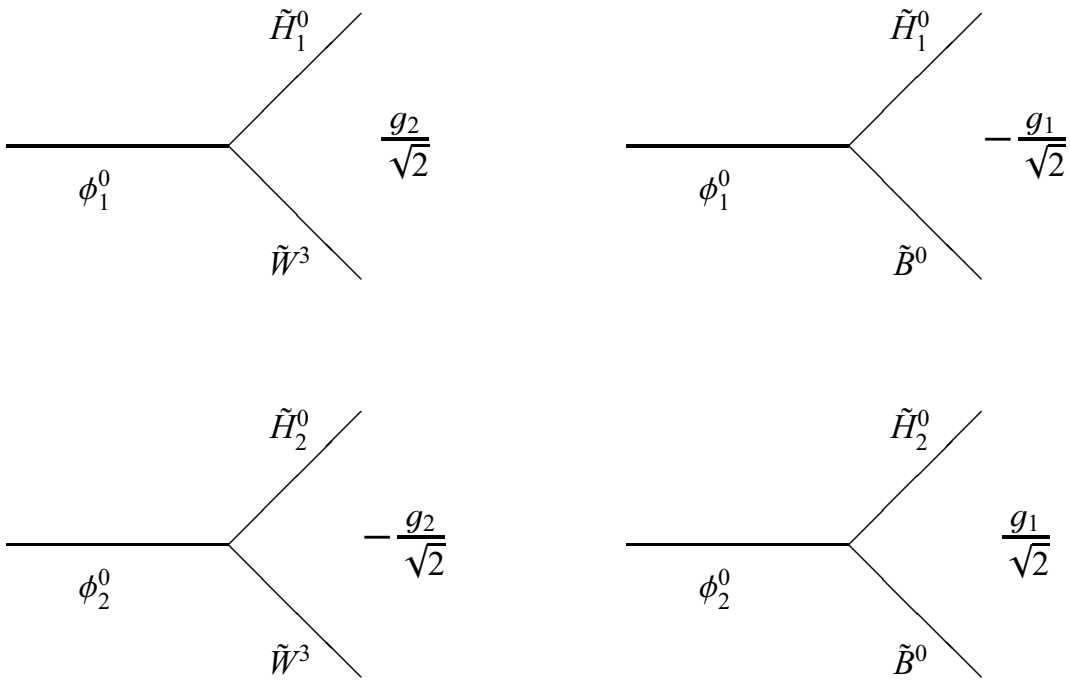

The general scheme of one-loop calculation is illustrated by the following example of $\lambda_{1}$ evaluation:
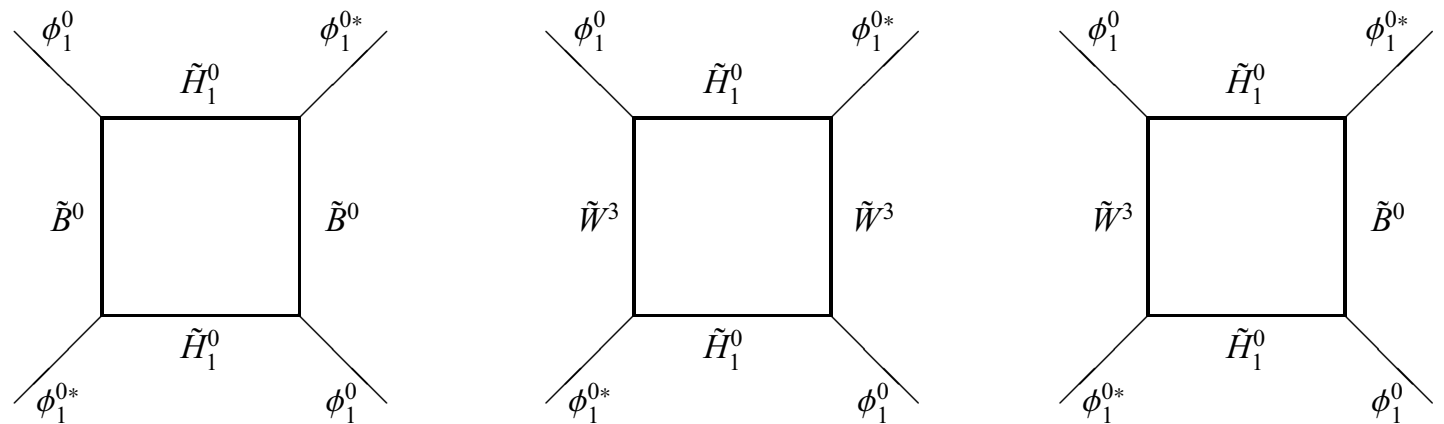

Calculation of all corrections at the one-loop gives the following result for the shift $\Delta \lambda_{i}$ (see $\lambda_{i}$ normalization in $[12,13])$ of the effective parameters

$$
-\frac{1}{2} \Delta \lambda_{1}=-\left(\frac{g_{1}^{4}}{4} J_{2}\left(m_{\tilde{H}_{1}^{0}}, m_{\tilde{B}^{0}}\right)+\frac{g_{2}^{4}}{4} J_{2}\left(m_{\tilde{H}_{1}^{0}}, m_{\tilde{W}^{3}}\right)+\frac{g_{1}^{2} g_{2}^{2}}{4} J_{3}\left(m_{\tilde{H}_{1}^{0}}, m_{\tilde{W}^{3}}, m_{\tilde{B}^{0}}\right)\right),
$$

where we have to deal with the above mentioned integrals over fermion loops. 


\section{Summary and Outlook}

Within the framework of the program the calculation technique is developed for one- and two-loop integrals in the finite-temperature field theory. The main object is the one-loop finite-temperature contribution to the mass state, had the same view in the form with the expression for the bosons ideal gas free energy. Principal object is the sum over Matsubara frequencies for relevant integrals allows representation in the form of generalized Hurwitz function. When masses are nondegenerated should further use operations with decomposition of rational fractions in the sum of (by analogy with Passarino-Veltman calculations methods), resulting in an arbitrary one-loop temperature diagram with several propagators expressed as derivatives of the basic object and rational fractions with itself. For the primary object there are well-known and well defined high-and low-temperature decompositions and renormalizability. Explicit submission as an infinite series or through the generalized Hurwitz function facilitate study of arbitrary graphs representing one-loop effective contributions to the potential. Conditions for the effective potential global and local minimums existence are included in the criteria for the validity of models with an extended Higgs sector [17, 18].

\section{References}

[1] R.P.Feynman, Phys.Rev.76:769-789,1949.

[2] A. J. Buras, P. H. Chankowski, J. Rosiek and L. Slawianowska, Nucl. Phys. B 659, 3 (2003) [arXiv:hep-ph/0210145].

[3] Athanasios Dedes, Janusz Rosiek, and Philip Tanedo, Phys.Rev. D 79, 055006 (2009).

[4] Dolgopolov M.V., Dubinin M.N., Rykova E.N. Two-Higgs doublet potential of the MSSM at finite temperature and Higgs boson masses // Quarks. 2008. V. 1.

[5] E. Akhmetzyanova, M. Dolgopolov, M. Dubinin, Self-couplings of Higgs bosons in scenarios with mixing of CP-even/CP-odd states, Workshop on CP Studies and Non-Standard Higgs Physics, CERN-2006-009 (May 2004 - December 2005), eds. S. Kraml, G. Azuelos, D. Dominici, J. Ellis, G. Grenier, H.,E. Haber, J. S. Lee, D. J. Miller, A., CERN, Geneva, 2006, 133138, arXiv: hep-ph/0608079.

[6] M. Dolgopolov, M. Dubinin, E. Rykova, J. Modern Phys., 2:5 (2011), 301-322.

[7] L. Vergara, Evaluating one loop integrals at finite temperature. Journal of Physics A30 (1997) 6977-6980

[8] P. Amore, Non perturbative regularization of one loop integrals at finite temperature. Journal of Physics A38 (2005) 6463-6472 (hep-th/0503142)

[9] P. Amore, Convergence acceleration of series through a variational approach. Journal of Mathematical Analysis and Applications 323 (2006) 63-77

[10] M. Abramowitz and I. Stegun, Handbook of Mathematical Functions, Dover Publications, NY, 1964

[11] E. Akhmetzyanova, M. Dolgopolov, M. Dubinin, Higgs bosons in the two-doublet model with CP violation. Phys.Rev. D71 (2005) 075008 (hep-ph/0405264)

[12] E. Akhmetzyanova, M. Dolgopolov, M. Dubinin, Violation of CP Invariance in the Two-Doublet Higgs Sector of the MSSM. Phys.Part.Nucl. 37 (2006) 677-734

[13] M.Dolgopolov, E.Rykova, M. Dubinin, I Erofeev Threshold corrections to the MSSM effective Higgs potential: gaugino and higgsino contributions // 20th International Workshop High Energy Physics and Quantum Field Theory, QFTHEP 2011, 24 September -1 October 2011, Sochi 2011, P. 1-8. 
[14] M. Laine, Effective theories of MSSM at high temperature. Nucl.Phys. B481 (1996) 43-84 (hepph/9605283); Erratum-ibid. B548: 637-638, 1999

[15] G.R. Farrar, M. Losada, SUSY and the electroweak phase transition. Phys.Lett. B406 (1997) 60-65 (hep-ph/9612346)

[16] M. Losada, High temperature dimensional reduction of the MSSM and other multiscalar models. Phys.Rev. D56 (1997) 2893-2913 (hep-ph/9605266)

[17] M. V. Dolgopolov, S. P. Zavodov, E. Yu. Petrova, Bifurcation sets of extended Higgs potential, Vestn. Samar. Gos. Tekhn. Univ. Ser. Fiz.-Mat. Nauki, 4(33) (2013), 173-183.

[18] M. N. Dubinin, E. Yu. Petrova, High-temperature Higgs potential of the two-doublet model in catastrophe theory, Teoret. Mat. Fiz., 184:2 (2015), 315-337. 